\title{
CONDORCET'S THEORY OF VOTING
}

\author{
H. P. YOUNG \\ University of Maryland
}

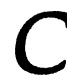

ondorcet's criterion states that an alternative that defeats every other by a simple majority is the socially optimal choice. Condorcet argued that if the object of voting is to determine the "best" decision for society but voters sometimes make mistakes in their judgments, then the majority alternative (if it exists) is statistically most likely to be the best choice. Strictly speaking, this claim is not true; in some situations Borda's rule gives a sharper estimate of the best alternative. Nevertheless, Condorcet did propose a novel and statistically correct rule for finding the most likely ranking of the alternatives. This procedure, which is sometimes known as "Kemeny's rule," is the unique social welfare function that satisfies a variant of independence of irrelevant alternatives together with several other standard properties.

[Our aim is] to inquire by mere reasoning, what degree of confidence the judgment of assemblies deserves, whether large or small, subject to a high or low plurality, split into several different bodies or gathered into one only, composed of men more or less wise.

- Condorcet

\section{A central problem}

in democratic theory is to justify the principle of majority rule. Why should the opinion of a majority of citizens or of elected representatives bind the rest of society? One of the earliest and most celebrated answers to this question was attempted by Rousseau in The Social Contract (1762). The opinion of the majority is legitimate, said Rousseau, because it expresses the "general will." "When a law is proposed in the people's assembly, what is asked of them is not precisely whether they approve of the proposition or reject it, but whether it is in conformity with the general will ... each by giving his vote gives his opinion on this question, and the counting of votes yields a declaration of the general will. When, therefore, the opinion contrary to my own prevails, this proves only that I have made a mistake, and that what I believed to be the general will was not so" (1962, 153).

This ambiguous and slightly disquieting idea was given a more satisfactory expression some twenty years later by the mathematician and social philosopher Marie Jean Antoine Nicolas Caritat, Marquis de Condorcet. ${ }^{1}$ In his remarkable work, Essai sur l'application de l'analyse à la probabilité des décisions rendues à la pluralité des voix (1785), Condorcet proposed the following argument for decision by majority: Enlightened voters honestly attempt to judge what decision will best serve society. They may occasionally judge wrongly. But assuming that they are more often right than wrong, the majority opinion will very likely be "correct." This is just a property of large numbers and is intuitively obvious when there are only two possible decisions. Condorcet rigorously demonstrated this proposition using the newly developed calculus of probabilities. He then proceeded to show how the idea could be elaborated into a whole series of results about the

\section{AMERICAN POLITICAL SCIENCE REVIEW VOL. 82 NO. 4 DECEMBER 1988}




\section{American Political Science Review Vol. 82}

reliability of decisions of a voting body, depending on its size, the competence of its members, and the number of alternatives under consideration. These "jury theorems" have recently attracted considerable attention (Grofman and Owen 1986; Grofman, Owen, and Feld 1983; Urken and Traflet 1984). ${ }^{2}$

It has not been widely recognized, however, that Condorcet showed how the same arguments could be extended to the case of more than two decisions. He was, of course, the first to realize the complications that arise in this case, namely, the possibility of cyclic majorities. But he went on to suggest-or at least came very close to suggesting-a definite rule for ranking any number of alternatives even when cyclic majorities are involved. Admittedly, Condorcet's argument is difficult to follow and full of inconsistencies. Moreover, the rule that he proposed is at variance with the algorithm that he proposed for computing it. Nevertheless, his stated goal is clear: to find the social decision that is most likely to be "correct."

First I summarize Condorcet's proposal and dispose of the claims made by some commentators that Condorcet gave no solution for the general case. I then show that Condorcet's method can be interpreted as a statistical procedure for estimating the ranking of the candidates that is most likely to be correct. If only one candidate is to be selected, however, then an earlier method proposed by Condorcet's rival Borda often gives better results. In the final section I show that Condorcet's decision rule can also be justified in modern social choice terms. It is the unique ranking procedure that satisfies a variant of independence of irrelevant alternatives together with several other standard conditions in social choice theory.

\section{Condorcet's Model}

For Condorcet, as for Rousseau, the object of voting is not merely to balance subjective opinions; rather, it is a collective quest for truth. In any field of candidates (or agenda of alternatives) there is some truly best candidate, a truly secondbest candidate, and so forth. What is meant here by truly best? Ultimately it is an undefined term, a notion that is postulated in order to make the theory worklike the ether in classical physics. To be sure, in some situations the notion of "best" decision is quite realistic. An example is trial by jury, where the group decision problem is to determine guilt or innocence. Another is the pooling of expert opinions about an observable event, like the state of the economy or tomorrow's weather. ${ }^{3}$

For the present let us grant Condorcet's hypothesis that the notion of a "correct" decision also applies to political decision making. Thus, voters evaluate candidates for political office in terms of their ability to lead. Legislators consider bills in terms of their potential contribution to the public welfare. Granted that voters are at least sometimes mindful of the public interest and vote accordingly, Condorcet's argument proceeds along the following lines. People differ in their opinions because they are imperfect judges of which decision really is best. If on balance each voter is more often right than wrong, however, then the majority view is very likely to identify the decision that is objectively best. In other words, the majority opinion gives the best estimate, in a statistical sense, of which candidate is most likely to be best. One of Condorcet's major contributions was to show how this idea can be generalized to the case of many candidates.

Consider a list of candidates (or decisions) that are voted upon pairwise. Assume that each voter, when faced with a comparison of the form, "Is $a$ better than $b$ or $b$ better than $a$ ?" chooses correctly with some fixed probability greater than $1 / 2$. Each voter judges every 
pair of candidates and the results are tallied in a voting matrix. Table 1 shows a voting matrix with 13 voters and three candidates. Each entry in the matrix gives the total number of votes obtained by the row candidate over the column candidate. For example, the comparison $a$ versus $b$ yields eight votes in favor of $a$, five in favor of $b$. The comparison $b$ versus $c$ yields eleven votes for $b$ and two for $c$. The comparison $a$ versus $c$ yields six for $a$ and seven for $c$. Notice that this vote entails the cyclical majority $a>b, b>c$, $c>a$.

Given a series of pairwise votes as in Table 1, the problem posed by Condorcet is to find the ranking of all the candidates that is most likely to be correct, that is, the ranking that would have produced the observed votes with highest probability. At first blush it might appear that this problem is insoluble unless the competency levels of the voters are known. This difficulty can be circumvented if one makes the simplifying assumption (which Condorcet did) that the voters all have the same competence. In this case the answer turns out to be independent of the level of competence, as I shall show in the next section. Here we shall content ourselves with Condorcet's statement of the solution.

First some terminology is required. In Condorcet's lexicon, an "opinion" is a series of pairwise comparisons on the alternatives. Each pairwise comparison is called a "proposition" and written $a>b$, etc. An opinion is said to be "impossible," "contradictory," or "absurd" if some of the propositions composing it form a cycle, such as $a>b, b>c, c>a$. Normally, each individual voter is able to rank all of the candidates in a consistent order. This is not necessarily true of the aggregate opinion, however, as Table 1 demonstrates. ${ }^{4}$ To break such cyclic majorities, Condorcet proposed the following method: ${ }^{5}$
Table 1. Voting Matrix with Three Alternatives and 11 Voters

\begin{tabular}{llll}
\hline & $a$ & $b$ & $c$ \\
\hline$a$ & - & 8 & 6 \\
$b$ & 7 & $\overline{2}$ & 11 \\
$c$ & 7 & & -
\end{tabular}

1. All possible opinions that do not imply a contradiction reduce to an indication of the order of merit that one judges to exist among the candidates .... therefore for $n$ candidates one would have $n(n-1) \ldots 2$ possible opinions.

2. Each voter having thus given his or her opinion by indicating the candidates' order of worth, if one compares them two by two, one will have in each opinion $n(n-1) / 2$ propositions to consider separately. Taking the number of times that each is contained in the opinion of one of the $q$ voters, one will have the number of voices who are for each proposition.

3. One forms an opinion from those $n(n-$ 1)/2 propositions that agree with the most voices. If this opinion is among the $n(n-1) \ldots$ 2 possible opinions, one regards as elected the subject to whom this opinion accords the preference. If this opinion is among the $2^{n(n-1) / 2}-$ $n(n-1) \ldots 2$ impossible opinions, then one successively deletes from that impossible opinion the propositions that have the least plurality, and one adopts the opinion from those that remain. (1785, 125-26)

Let us apply this rule to the situation in Table 1. First we are to choose the three propositions having a majority, namely $b$ $>c$ with eleven votes, $a>b$ with eight votes, and $c>a$ with seven votes. Since these three propositions form a cycle, however, we delete the proposition with smallest plurality, namely $c>a$. This leaves the combination $b>c$ and $a>b$ (and implicitly $a>c$ ), which implies the ranking $a>b>c$.

The rule is clear enough in the case of three alternatives. However, in the general case some ambiguities arise. The difficulty lies in the phrase "successively deletes" in step 3. Taken literally, it means the following: If there are cycles in the propositions selected in step 2, delete first the proposition having the least number of votes in its favor. (This proposition 


\section{American Political Science Review Vol. 82}

Table 2. A Voting Matrix with 25 Voters and Four Alternatives

\begin{tabular}{lrrrr}
\hline & $a$ & $b$ & $c$ & $d$ \\
\hline$a$ & - & 12 & 15 & 17 \\
$b$ & 13 & - & 16 & 11 \\
$c$ & 10 & 9 & - & 18 \\
$d$ & 8 & 14 & 7 & - \\
\hline
\end{tabular}

might or might not be contained in a cycle.) If any cycles remain, delete next the proposition with the next lowest number of votes in its favor, and so forth. Continue until all cycles are eliminated.

There are several reasons why this is probably not what Condorcet meant to say. In the first place, the procedure may yield an ambiguous ordering of the candidates. Consider Table 2, with twenty-five voters and four candidates.

In step 2 of Condorcet's algorithm one would select the six propositions having greatest majorities. In descending size of majority, these are $c>d, a>d, b>c, a$ $>c, d>b, b>a$. According to a literal reading of step 3 , one would first delete the proposition $b>a$, as it has the smallest majority in its favor. But this does not result in an "opinion" because one cycle still remains: $b>c, c>d, d>b$. Therefore one would delete the proposition $d>$ $b$, as it has the next-smallest majority in its favor. All cycles are now eliminated. But there is a difficulty: in the resulting partial order both $a$ and $b$ are undominated. Either one of them could be interpreted as the top-ranked candidate, so the outcome is indeterminate.

It seems more likely that Condorcet meant to reverse, rather than simply delete, the weakest propositions. According to this interpretation one would first reverse the proposition with the least majority (thus $b>a$ becomes $a>b$ ), then reverse the proposition with the next-least majority (thus $d>b$ becomes $b$ $>d)$ and so forth until no cycles remain.
In the above example the resulting opinion is abcd. Unfortunately, this answer does not square with the rest of Condorcet's argument. His stated intention is to choose "the most probable combination of opinions," that is, the set of propositions that contains no cycles and is supported by the largest number of pairwise votes. The total number of votes supporting the ranking abcd is 89. (That is, the sum of the votes for $a$ over $b, a$ over $c$, $a$ over $d, b$ over $c, b$ over $d$, and $c$ over $d$ equals 89.) But this is not a maximum. The ranking bacd is supported by a total of 90 pairwise votes. This ranking is obtained by reversing exactly one majority proposition, $d>b$. Note that this is not the proposition with smallest majority.

At this point we might be tempted to throw up our hands and declare, as previous commentators have, that "the general rules for the case of any number of candidates as given by Condorcet are stated so briefly as to be hardly intelligible ... and as no examples are given it is quite hopeless to find out what Condorcet meant" (E. J. Nanson as quoted in Black 1958, 175). Even the influential mathematician Isaac Todhunter found Condorcet's argument completely exasperating: "The obscurity and self-contradiction are without any parallel, so far as our experience of mathematical works extends ... no amount of examples can convey an adequate impression of the evils" (Todhunter 1949, 352).

In fact, Condorcet's overall intention is quite clear. It is to find the most probable combination of opinions. In describing his method he seems to have used the term "successively deletes" rather loosely as a heuristic for computing the solution. ${ }^{\circ}$ ( $\mathrm{He}$ may also have believed, mistakenly, that successive deletion always does give the most probable combination.) But there appears to be little doubt about his objective. Hence I propose to amend Condorcet's statement of the rule as follows: 
$3^{\prime}$. One forms an opinion from those $n(n-1) / 2$ propositions that agree with the most voices. If this opinion is among the $n(n-1) \ldots 2$ possible opinions, one regards as elected the subject to whom this opinion accords the preference. If this opinion is among the $2^{n(n-1) / 2}-n(n-1) \ldots 2$ impossible opinions, then one reverses in that impossible opinion the set of propositions that have the least combined plurality and one adopts the opinion from those that remain.

It seems reasonably likely that this is what Condorcet meant to say. In any case, it is the only interpretation that is consistent with his goal of finding the ranking that is most likely to be correct.

\section{Condorcet's Method As a Statistical Hypothesis Test}

From a historical standpoint, Condorcet's theory is particularly interesting because it represents one of the earliest applications of what today would be called "statistical hypothesis testing." Based on a sample of observations, one wishes to infer which of several underlying but unobservable states of nature is most likely to be true. This approach is known as maximum likelihood estimation. All that is required is a model of how the observed quantities depend probabilistically on the unobservable state of nature. In Condorcet's scheme of things a state of nature corresponds to the true or correct ordering of the candidates. This datum is essentially unobservable. What can be observed are the votes of the representatives who are attempting to read the true state of nature.

Condorcet assumed, first, that in any pairwise comparison each voter will choose the better candidate with some fixed probability $p$, where $1 / 2<p<1$ and $p$ is the same for all voters. ${ }^{7}$ Second, every voter's judgment on every pair of candidates is independent of his or her judgment on every other pair. Finally, each voter's judgment is independent of the other voters' judgments (a significant assumption).
Consider again the voting matrix in Table 1. Suppose that the true ranking is $a b c-a$ first, $b$ second, $c$ third. A vote for $a$ over $b$ will occur with probability $1-$ $p$, whereas a vote for $b$ over $a$ will occur with the lesser probability $1-p$. The combined probability of observing the 39 pairwise votes in Table 1 is therefore

$$
\begin{aligned}
& L(a b c)=\frac{13 !}{8 ! 5 !}\left[p^{8}(1-p)^{5}\right] \\
& \frac{13 !}{6 ! 7 !}\left[p^{6}(1-p)^{7}\right] \frac{13 !}{11 ! 2 !}\left[p^{11}(1-p)^{2}\right] \\
& =\frac{(13 !)^{3}}{8 ! 5 ! 6 ! 7 ! 11 ! 2 !}\left[p^{25}(1-p)^{14}\right] .
\end{aligned}
$$

Similarly, the probability of observing the results in Table 1 if the true ranking is $a c b$ would be

$$
\begin{aligned}
& L(a b c)=\frac{13 !}{8 ! 5 !}\left[p^{8}(1-p)^{5}\right] \\
& \frac{13 !}{6 ! 7 !}\left[p^{6}(1-p)^{7}\right] \frac{13 !}{11 ! 2 !}\left[p^{2}(1-p)^{11}\right] \\
& =\frac{(13 !)^{3}}{8 ! 5 ! 6 ! 7 ! 11 ! 2 !}\left[p^{16}(1-p)^{23}\right]
\end{aligned}
$$

The coefficient is the same for all six rankings, so their relative likelihoods are as in Table 3.

For each ranking the exponent of $p$ is the sum of all pairwise votes that agree with the ranking, and the exponent of (1 $-p$ ) is the sum of all pairwise votes that disagree with it. Assuming that $p>1 / 2$,

Table 3. Likelihoods of Six Rankings Based on Table 1 Data

\begin{tabular}{cc}
\hline \hline Ranking & Likelihood \\
\hline$a b c$ & $p^{25}(1-p)^{14}$ \\
$a b c$ & $p^{16}(1-p)^{23}$ \\
$b a c$ & $p^{22}(1-p)^{17}$ \\
$b c a$ & $p^{23}(1-p)^{16}$ \\
$c a b$ & $p^{17}(1-p)^{22}$ \\
$c b a$ & $p^{14}(1-p)^{25}$ \\
\hline
\end{tabular}




\section{American Political Science Review Vol. 82}

which seems to be a credibly optimistic view of human judgment, it follows that the most likely ranking is the one with the highest exponent of $p$. In the above example the answer is $a b c$.

In general, suppose that there are $n$ voters and $m$ candidates or alternatives $a_{1}, \ldots, a_{m}$. Let $n_{i j}$ be the total number of votes that $a_{i}$ receives over $a_{j}$. Assume that every voter registers an opinion on every pair of candidates, so that $n_{i j}+n_{i i}=n$ for all $i \neq j$. Let $\mathrm{R}$ be a complete ranking of the candidates, where $a_{i} R a_{j}$ means that $a_{i}$ is preferred to $a_{j}$. The argument outlined above is easily generalized to show that a ranking $R$ has maximum likelihood if and only if it maximizes $\Sigma n_{i j}$, the sum over all pairs $i j$ such that $a_{i} R a_{i}$. That is, the maximum likelihood ranking is the one that agrees with the maximum number of pairwise votes, summed over all pairs of candidates. This is equivalent to the statement of Condorcet's rule given in $3^{\prime}$. Note that it does not depend on the value of the success rate $p$ as long as all voters have the same success rate, which is larger than one half. This rule is very natural and, not surprisingly, has been rediscovered by other writers. In particular it was proposed in a different guise by John Kemeny (1959; see also Kemeny and Snell 1960) and is sometimes known in the literature as "Kemeny's rule" (Young and Levenglick 1978).

\section{Condorcet versus Borda}

Another pioneer in the theory of elections was the Chevalier Jean Charles de Borda. Like Condorcet, Borda was a member of the French Academy of Sciences and an important figure in official scientific circles. Unlike Condorcet, he was an applied scientist with a practical bent. He was noted for his work in hydraulics, mechanics, optics, and especially for the design of advanced navigational instruments. He also captained maritime vessels. Because of his ex- pertise in measurement, he served on the committee for determining the standard weights and measures in the new metric system (Mascart 1919).

In 1770 , some 15 years prior to the publication of Condorcet's treatise on elections, Borda read a paper to the academy on the design of voting procedures (see Borda 1784). Exactly what prompted Borda to sail in these uncharted waters is unclear. Possibly he was concerned with reforming the election procedures in the academy itself. He began by pointing out the defects with the customary "first-pastthe-post" system in which the candidate with the largest plurality is elected. When there are many candidates, this procedure can easily elect someone who is endorsed by only a small minority of the electorate. Instead, Borda suggested, the election procedure should be based on each voter's entire rank ordering of the candidates. The correct candidate to choose is the one that on average stands highest in the voters' lists.

Operationally, the method works as follows: Each voter submits a list in which all of the candidates are rank-ordered. In each list a candidate who is ranked last receives a score of zero, a candidate who is ranked next-to-last receives a score of one, a candidate who is ranked next above that receives a score of two, and so forth. Thus the score of a candidate in any particular list is equal to the number of candidates ranked lower on that list. The scores of the candidates are summed over all voter lists and the candidates with highest total score is elected.

If Borda's objective was practical reform, he appears to have been successful, for something like his method was subsequently adopted for the election of new members to the academy. ${ }^{8}$

Condorcet was certainly aware of Borda's work. Indeed he introduced the method in the Essai with the following remark: "As the celebrated Geometer to whom one owes this method has pub- 
lished nothing on this subject, I have taken the liberty of citing it here" (1785, clxxix). ${ }^{9}$ Condorcet repeatedly refers to Borda, not by name, but as "the celebrated Geometer." "The tone is ironical. From his private correspondence it is clear that Condorcet held Borda's work in low esteem and considered him a personal enemy within the academy. For example, in a letter to Turgot (1775), he gave this assessment: "[M. Malesherbes] makes a great case for Borda, not because of his memoirs, some of which suggest talent (although nothing will ever come of them, and no one has ever spoken of them or ever will) but because he is what one calls a good academician, that is to say because he speaks in meetings of the Academy and asks for nothing better than to waste his time doing prospectuses, examining machines, etc., and above all because, feeling eclipsed by other geometers, he, like d'Arcy, has abandoned geometry for petty physics" (quoted in Henry 1883). The contempt with which Condorcet viewed Borda's scientific contributions may help to clarify an abrupt shift in Condorcet's argument. As we have already seen, Condorcet's initial objective was to design a method for estimating the "true" ranking of the candidates. But he also recognized that the problem is often one of determining which single candidate is most likely to be best (1785, lxi-lxv, 12223). At first glance the solution would appear to be obvious: first rank all of the candidates and then choose the one that is top-ranked. But this is not necessarily the most convincing answer.

Consider the following example of Condorcet (1785, lxiii) with 60 voters and three candidates. Here $c$ has a simple majority over both $a$ and $b$ and $b$ has a simple majority over $a$. So there are no cycles, and the ranking by Condorcet's rule is cba. That is, $c b a$ is the ranking most likely to be correct. But is $c$ the candidate that is most likely to be the best (i.e., top-ranked in the true ranking)?
Table 4. A Voting Matrix with 60 Voters and Three Candidates

\begin{tabular}{cccc}
\hline \hline & $a$ & $b$ & $c$ \\
\hline$a$ & - & 23 & 29 \\
$b$ & 37 & - & 29 \\
$c$ & 31 & 31 & - \\
\hline
\end{tabular}

Not necessarily. Candidate $c$ will be best if two propositions hold: namely, if $c$ is better than $a(c>a)$ and $c$ is better than $b$ $(c>b)$. We are therefore interested in the joint probability of these two statements being true. If $c>a$, then the probability of observing the voting pattern in Table 4 (31 votes for $c$ versus 29 for $a$ ) is $(60 ! / 31 ! 29 !) p^{31}(1-p)^{29}$. If the contrary holds $(a>c)$, then the probability of observing 31 votes for $c$ versus 29 for $a$ is $(60 ! / 31 ! 29) p^{29}(1-p)^{31}$. Assume that $a>$ $c$ and $c>a$ are a priori equally likely. Then the probability of observing the vote 31 for $c, 29$ for $a$ is

$$
\begin{aligned}
\operatorname{Pr}(\text { vote }) & =(60 ! / 31 ! 29 !)\left(p^{31}(1-p)^{29}\right. \\
& \left.+p^{29}(1-p)^{31}\right) / 2 .
\end{aligned}
$$

By Bayes' rule ${ }^{10}$ it follows that the posterior probability of $c>a$, given the observed vote, is

$$
\begin{aligned}
& \operatorname{Pr}(c>a \mid \text { vote })= \\
& \frac{\operatorname{Pr}(\text { vote } \mid c>a) \operatorname{Pr}(c>a)}{\operatorname{Pr}(\text { vote })} .
\end{aligned}
$$

that is,

$$
\begin{aligned}
& \operatorname{Pr}(c>a \mid \text { vote })= \\
& \frac{p^{31}(1-p)^{29}}{p^{31}(1-p)^{29}+p^{29}(1-p)^{31}} .
\end{aligned}
$$

Similarly,

$$
\begin{aligned}
& \operatorname{Pr}(c>b \mid \text { vote })= \\
& \frac{p^{31}(1-p)^{29}}{p^{31}(1-p)^{29}+p^{29}(1-p)^{31}} .
\end{aligned}
$$




\section{American Political Science Review Vol. 82}

Therefore the posterior probability that $c$ is best is

$$
\begin{aligned}
& \operatorname{Pr}(c \mid \text { vote })= \\
& \quad \operatorname{Pr}(c>a \mid \text { vote }) \operatorname{Pr}(c>b \mid \text { vote })= \\
& \frac{\left[p^{31}(1-p)^{29}\right]^{2}}{\left[p^{31}(1-p)^{29}+p^{29}(1-p)^{31}\right]^{2}} .
\end{aligned}
$$

The posterior probability that $b$ is best is

$$
\begin{aligned}
& \operatorname{Pr}(b \mid \text { vote })= \\
& \frac{p^{37}(1-p)^{23} p^{29}(1-p)^{31}}{\left[p^{37}(1-p)^{23}+p^{23}(1-p)^{37}\right] \times}
\end{aligned}
$$

And the posterior probability that $a$ is best is

$$
\begin{aligned}
& \operatorname{Pr}(a \mid \text { vote })= \\
& \frac{p^{23}(1-p)^{37} p^{29}(1-p)^{31}}{\left[p^{23}(1-p)^{37}+p^{37}(1-p)^{23}\right] \times} \\
& \left.\left.\quad+p^{31}(1-p)^{29}\right]\right\} .
\end{aligned}
$$

Which of $a, b$, or $c$ has the highest posterior probability? The answer depends on the assumed value of the competence level $p$. If $p$ is close to one, then the controlling factor is the size of the exponent of $1-p$. The most probable candidate will be the one whose weakest comparison is as strong as possible. ${ }^{11}$ In this case the answer would be $c$, because the lowest number of votes that $c$ receives in any pairwise contest is 31 , while $a^{\prime}$ s weakest showing is 23 and $b^{\prime}$ s is 29 .

Suppose instead that $p$ is close to $1 / 2$, that is, $p=1 / 2+\epsilon$ for some small $\epsilon>0$. In this case the relative probabilities depend essentially only on the numerators of the above three expressions. Substituting in $p=1 / 2+\epsilon$ we find that the relative probabilities of $a, b$, and $c$ can be approximated as follows:

$$
\begin{aligned}
& \text { a: }(1 / 2+\epsilon)^{23}(1 / 2-\epsilon)^{37}(1 / 2+\epsilon)^{29}(1 / 2-\epsilon)^{31} \\
& \text { b: }(1 / 2+\epsilon)^{37}(1 / 2-\epsilon)^{23}(1 / 2+\epsilon)^{29}(1 / 2-\epsilon)^{31} \\
& \text { c: }(1 / 2+\epsilon)^{31}(1 / 2-\epsilon)^{29}(1 / 2+\epsilon)^{31}(1 / 2-\epsilon)^{29}
\end{aligned}
$$

If we expand these expressions using the binomial formula and drop all powers of $\epsilon$ higher than the first, then the relative probabilities of the three candidates are approximately as follows:

$$
\begin{aligned}
& \text { a: }(1 / 2)^{120}+(1 / 2)^{119}(23-37+29-31) \epsilon \\
& \text { b: }(1 / 2)^{120}+(1 / 2)^{119}(37-23+29-31) \epsilon \\
& \text { c: }(1 / 2)^{120}+(1 / 2)^{119}(31-29+31-29) \epsilon
\end{aligned}
$$

In each case the coefficient of $\epsilon$ is the sum of all pairwise votes for the candidate minus the sum of all pairwise votes against the candidate. Since the total number of votes (for and against) is the same for all candidates, the coefficient of $\epsilon$ will be maximized for that candidate that receives the most pairwise votes over all others. In this case the answer is $b$.

This argument is quite general. For any number of voters and any number of alternatives, if $p$ is sufficiently close to $1 / 2$, then the candidate with the highest probability of being best is the one that receives the most pairwise votes. This is precisely the Borda candidate. The reason is that the Borda score of a candidate in each individual voter's list is equal to the number of candidates ranked below it, that is, the number that it beats. So a candidate's total Borda score is its row sum in the voting matrix. ${ }^{12}$

Borda's method is therefore one solution to Condorcet's problem. In particular, a candidate that beats every other by simple majority does not necessarily have the strongest claim to being the best candidate; the Borda winner may be stronger. ${ }^{13}$ Condorcet apparently realized this implication of his argument. Understandably, he would be loath to draw much attention to it, given his feelings toward Borda. In addition, Condorcet may have become disillusioned with his approach because of its failure to provide a simple and definitive solution independently of the competence level $p$.

For whatever reason, Condorcet abruptly changed course at this point in the Essai (1785, lxiii-lxv). He abandoned the statistical framework that he had so painstakingly built up and fell back on a more "straightforward" line of reasoning. In doing so he opened up a whole new 


\section{Condorcet's Theory}

approach that has had enormous influence on the modern theory of social choice. To illustrate Condorcet's newfound argument, consider the example in Table 4. If a candidate, such as $c$, obtains a simple majority over every other candidate then, Condorcet argued, that candidate is the only reasonable choice. Indeed, candidate $a$ has no reasonable claim to being best, therefore $a$ should be set aside. So the contest is between $b$ and $c$. But $c$ defeats $b$, so the choice must come down on the side of $c$.

More generally, Condorcet proposed that whenever a candidate obtains a simple majority over every other candidate, then that candidate is presumptively the "best." This decision rule is now known as "Condorcet's criterion," and such a candidate (if it exists) is a "Condorcet winner" or a "majority candidate" (Fishburn 1973).

In advancing this new approach, Condorcet was not abandoning his basic premise that the object of voting is to make the "correct" choice. He merely shifted his ground and argued for adopting a simple estimate of the best choice rather than a complex one. Furthermore, this "new" approach is entirely consistent with Condorcet's earlier solution of the ranking problem. Indeed, if a majority candidate exists, then it must be ranked first in a Condorcet ranking. The reason is simple. Suppose that $x$ is a Condorcet candidate but that this candidate is not ranked on top in the Condorcet ranking. Then it must be ranked just below some other candidate $y$. By assumption, $x$ defeats $y$ by a simple majority. Therefore, if we switch only the order of $x$ and $y$ in the ranking, then we obtain a new ranking that is supported by more pairwise votes. But this contradicts the definition of the Condorcet ranking as the one supported by the maximum number of pairwise votes. Thus Condorcet's choice rule is consistent with his ranking rule whenever a majority candidate exists.
While Condorcet's solution is attractive, Borda's rule still has advantages that cannot be dismissed easily. First, it is considerably simpler to compute than Condorcet's ranking rule. Second, if one accepts Condorcet's basic premises, then the Borda winner is in fact a better estimate of the best candidate provided that $p$ is close to $1 / 2$. Third, if $p$ is not close to $1 / 2$, then it is still very likely that the Borda winner is the best candidate, even though strictly speaking it may not be the optimum estimate of the best candidate. ${ }^{14}$ If there are a large number of voters and $p$ is not very close to $1 / 2$, then the probability is very high that the truly best candidate will be selected by any reasonable choice rule (i.e., with high probability it will be the majority winner and the Borda winner at the same time). The critical case is precisely when $p$ is near $1 / 2$, and then Borda's rule is definitely optimal. Thus we must either jettison the whole idea of selecting the "best" candidate with high probability or admit that Borda's rule is a good way of estimating which candidate that is.

On the other hand, when the problem is to rank a set of alternatives, Condorcet's rule is undoubtedly better than Borda's. Furthermore, even if one does not accept the specific probability model on which this conclusion is based, Condorcet's rule has an important stability property that Borda's rule lacks, as I shall now show.

\section{Local Stability and Nonmanipulability}

One of the most important concepts in the theory of social choice is independence: a social decision procedure should depend only on the voters' preferences for the "relevant" alternatives, not on their preferences for alternatives that are infeasible or outside the domain of discourse. Independence has been given several distinct formulations (Arrow 1963; 
Table 5. A Voting Matrix on Six Alternatives and 100 Voters

\begin{tabular}{|c|c|c|c|c|c|c|c|}
\hline & $a$ & $b$ & $c$ & $d$ & $e$ & $f$ & Borda Scores \\
\hline$a$ & - & 51 & 54 & 58 & 60 & 62 & 2 \\
\hline$b$ & 49 & - & 68 & 56 & 51 & 58 & 283 \\
\hline$c$ & 46 & 32 & - & 70 & 66 & 75 & 289 \\
\hline$d$ & 42 & 44 & 30 & - & 41 & 64 & 221 \\
\hline$e$ & 40 & 48 & 34 & 59 & - & 34 & 215 \\
\hline$f$ & 38 & 42 & 25 & 36 & 66 & - & 207 \\
\hline
\end{tabular}

Nash 1950). The one that I shall adopt here is closest in spirit to Nash's version: if the set of alternatives shrinks, then the reduced set of alternatives should be ranked in the same way that they were within the larger ranking. There are at least two reasons why independence is desirable. First, it says that decisions cannot be manipulated by introducing extraneous alternatives. Second, it allows sensible decisions to be made without requiring an evaluation of all possible decisions. In practice, society cannot consider all possible choices in a situation. It cannot, or at least usually does not, even consider all of the best choices. In choosing a candidate for public office, for example, it is impractical to consider all eligible citizens. In considering a piece of legislation, it is impossible to hold votes on all possible amendments. Independence assures that a decision made from a limited agenda of alternatives is valid relative to that agenda. Enlarging the agenda may introduce better possibilities, but it should not cause us to revise the relative ranking of the old possibilities.

Unfortunately, it is impossible to design any reasonable social decision rule with this property. I claim, however, that independence can be satisfied if we restrict ourselves to agendas that are sufficiently "connected." By a connected agenda I mean, roughly speaking, a subset of closely related alternatives rather than an arbitrary collection of unrelated or extreme ones. Consider the following example: $\mathrm{A}$ legislature of 100 members is considering three alternative decisions $a, b$, and $c$ (see Table 5). Other alternatives might in theory be considered, such as $d, e$, and $f$, but they are weaker than $a, b$, and $c$ in the sense that any one of them would be defeated by any one of $a, b$, or $c$.

The question is whether the exclusion of the nominally "inferior" alternatives $d$, $e, f$ affect the choice between $a, b$, and $c$. Put differently, can an inferior alternative be introduced strategically in order to manipulate the choice between the "superior" alternatives? This is surely a situation that we would like to avoid. Unfortunately Borda's rule suffers from this defect. To see this, observe that the total Borda score of each alternative is simply the sum of the row entries corresponding to that alternative (e.g., the right-hand column of Table 5). These scores imply the ranking cabdef. Now consider just the three alternatives $a, b$, and $c$. The corresponding voting matrix is the one enclosed by dashed lines. The Borda scores for this three-alternative situation are 117 for $b, 105$ for $a$, and 78 for $c$. Hence when just the top three alternatives are considered, they would be ordered bac, which is the reverse of how the electorate would have ordered them in the context of all alternatives. Introducing the inferior alternatives changes the outcome from $b$ to $c$.

I claim that Condorcet's rule is immune 
to this type of instability. To compute the Condorcet solution observe first that $a$ has a simple majority over every other alternative, hence (as was demonstrated in the previous section) Condorcet's rule must rank $a$ first. Since each of the alternatives $a, b$, and $c$ defeats each of the alternatives $d, e$, and $f$ by a majority, it also follows that the former three alternatives must be ranked above the latter three. For if this were not the case, then some alternative would be ranked immediately below another alternative that it defeats, which is impossible in a Condorcet ranking. To maximize agreement with the pairwise voting data, $a$ must be ranked first, $b$ second, and $c$ third. Positions four, five, and six in the ranking will be occupied by $d, e$, and $f$ in some order. To determine which order, it suffices to apply Condorcet's criterion to these three alternatives alone; that is, they should be ordered so as to agree with the maximum number of pairwise votes that they receive among themselves. The reader may verify that the ordering that accomplishes this objective is dfe. Hence the total ordering implied by Condorcet's rule is abcdfe. This is quite different from the Borda ordering.

I have worked out the details of this example to show that Condorcet's rule is not necessarily difficult to compute even when a sizable number of alternatives is involved (though it is certainly more difficult than Borda's method). It also illustrates why Condorcet's method satisfies a certain form of independence. For example, if the bottom three alternatives are dropped from consideration, then Condorcet's rule (unlike Borda's) must preserve the ordering $a b c$. Indeed, if it did not, then the interval $a b c$ could be rearranged into a new ordering that is supported by more pairwise votes. But then $a b c$ could also be rearranged within the full ordering abcdfe to obtain a new ordering that is supported by more pairwise votes. This contradicts the assump- tion that abcdfe is supported by the maximum number of pairwise votes. A similar argument applies if the last two alternatives are dropped, the last four, or indeed any bottom segment of the ranking. Similarly, the ordering of the bottom part of the list will be preserved if we remove the top alternative, the top two alternatives, or any top segment of the ranking.

In general, a Condorcet ranking has the property that the ordering of the alternatives does not change whenever we restrict attention to an interval of the full ordering. ${ }^{15}$ This property will be called local stability. Intervals tend to consist of alternatives that are relatively closely related. For example, an interval might consist of a set of candidates that occupy a certain segment of the political spectrum (e.g., "centrist"). Or it might consist of a series of minor modifications or amendments to a proposed piece of legislation.

I shall show that Condorcet's method is the only plausible ranking procedure that is locally stable. To do so we must recall several properties that are standard in the social choice literature. Let $F$ be a social ranking rule, that is, a rule for strictly ordering any set of alternatives given the preference data of the voters. We shall assume that the input data to $F$ is given in the form of a voting matrix: the number of votes for and against each pair of alternatives. All voters are weighted equally because only the number of votes counts, not the identities of the individuals who cast the votes. This property is known in the literature as anonymity. $F$ is neutral if it is not "biased" with respect to one or another alternative: if the names of the alternatives are permuted, then their ranking is similarly permuted. Taken together, anonymity and neutrality require that $F$ sometimes yields ties. ${ }^{16} \mathrm{~A}$ third standard condition in the social choice literature is that $F$ be unanimous: if all voters cast their votes the same way on every pair, then the ordering of the candidates agrees with all of the votes. 


\section{American Political Science Review Vol. 82}

Finally we shall require a degree of consistency in the way that $F$ aggregates individual opinions into group opinions. Consider a bicameral system such as the U.S. Congress. To pass, a decision must receive a majority of both chambers. This much is clear when a decision involves just two choices. Now consider a decision involving more than two alternatives. Suppose that both chambers use the same decision rule $F$, and that they consider the same agenda. Let each chamber separately rank all of the alternatives. If both chambers happen to rank them in exactly the same way, then we may say that this ranking is approved by both chambers. If a ranking is approved by both chambers, then it stands to reason that it would also be approved if the two chambers were to be considered as a single body (i.e., to cast their votes in plenary session). $F$ satisfies reinforcement if whenever a ranking is approved by two separate groups of voters, then it would also be approved when the votes of the two groups are pooled. ${ }^{17}$

The condition of "local stability" can now be formally stated as follows: Let $A$ be an agenda of alternatives, let $V$ be a voting matrix on $A$, and let $F(V)=R$ be the (unique) consensus ranking. $F$ is locally stable if for every interval $B$ of the ranking $R, F\left(V_{B}\right)=R_{B}$ where $V_{B}$ and $R_{B}$ represent $V$ and $R$ restricted to the subset $B$. In case of ties, the condition says that for every $R \epsilon F(V)$, every interval $B$ of $R, R_{B} \epsilon$ $F\left(V_{B}\right)$, and if $R_{B}^{\prime} \in F\left(V_{B}\right)$ for some $R_{B}^{\prime} \neq$ $R_{B}$, then $R_{B}^{\prime}$ can be substituted for $R_{B}$ in $R$ to obtain a new ranking $R^{\prime} \in F(V)$.

It follows from a theorem of myself and Arthur Levenglick (1978) that Condorcet's rule is the unique ranking rule that satisfies anonymity, neutrality, unanimity, reinforcement, and local stability. ${ }^{18}$ Note that Borda's rule satisfies all of these properties except local stability.

\section{Conclusion}

The purpose of this article has been to draw attention to a major, but little- known, contribution by Condorcet to the theory of group decision making. Condorcet proposed a decision rule that applies to any voting situation whether cyclic majorities are present or not. This rule can be interpreted as a best guess about the "true" ordering of the alternatives. The rule can also be justified on entirely diffeent grounds; namely, it is unbiased with respect to both individuals and alternatives and it aggregates individual opinions in a "consistent" way. This axiomatic justification differs significantly from the probabilistic one in that it places emphasis on the operational properties of the method rather than on a model about how the world works. Of course one may still question whether these are the "right" properties, just as one may question whether Condorcet had the "right" probabilistic model. But at least the nature of the choice is clarified. Furthermore, one must admit that the specific probabilistic model by which Condorcet reached his conclusions is almost certainly not correct in its details. The plausibility of his solution must therefore be subjected to other tests, as I have attempted to do.

I conclude that there is a strong case for Condorcet's rule on two counts. First, in situations where voters are primarily concerned with the accuracy of a decision (as in jury trials, expert opinion pooling, and in some types of public referenda), Condorcet's method gives the ranking of the outcomes that is most likely to be correct. Second, where voters are motivated primarily by their subjective preferences about outcomes, Condorcet's rule is responsive to changes in individual preferences, is unbiased with respect to voters and alternatives, and (unlike Borda's rule) it is relatively stable against manipulation by the introduction of unrealistic or inferior alternatives. In short, we need not resolve the question whether voters attempt to judge which decision is in the public interest or whether they merely register their subjective opinions on an issue. Both of these elements are probably present in most decisions. In either case, 
Condorcet's method is a rational way of aggregating individual choices into a collective preference ordering.

\section{Notes}

This work was supported by the Office of Naval Research under contract N00014-86-K-0586 at the University of Maryland. The author is indebted to Bernard Grofman and William Riker for very helpful comments on the manuscript.

1. Condorcet did not explicitly say that he was addressing Rousseau's problem, but Rousseau's ideas would have been much discussed in Condorcet's circles. Baker $(1975,229)$ also suggests that Condorcet drew his inspiration from Rousseau. Grofman and Feld (1988) explore the connections between Condorcet's and Rousseau's ideas in greater depth.

2. Poisson (1837) studied a similar set of questions using probabilistic methods. See also Gelfand and Solomon (1973) for recent work in the Poisson tradition.

3. A related problem is to rank teams or players based on their win-loss records (as in baseball, tennis, or chess). Condorcet's model can be used to estimate the most likely true ordering of the players (see Batchelder and Bershad 1979; Good 1955; Jech 1983).

4. The following preference data would yield the pairwise votes in Table 1: six voters have preference $a b c$, five voters have preference $b c a$, and two voters have preference $c a b$. It bears emphasizing, however, that Condorcet did not think of the voters' opinions as expressing their personal preferences. Rather, they represent the voters' best judgments as to which candidate in each pair is the better one.

5 . In this translation I have modified the mathematical notation slightly to conform with modern conventions. sion.

6. Michaud (1985) comes to a similar conclu-

7. I exclude the case $p=1$, because this would imply that all voters agree with probability one. The case where the voters have different a priori probabilities of being right is treated in Young 1986 (see also Nitzan and Paroush [1982] and Shapley and Grofman [1984]).

8. For reasons that remain obscure, Borda's method was jettisoned by Napoleon after his own election to membershp (Mascart 1919).

9. In a footnote, Condorcet adds that his own treatise "had been printed in its entirety before I had any acquaintance with this method [of Borda], apart from the fact that I had heard several people speak of it" (1785, clxxix).
10. Condorcet arrived at this answer intuitively, not by an explicit application of Bayes' rule.

11. This "minimax" rule was proposed in modern times by Kramer (1977).

12. Borda himself gave this alternative statement of his method.

13. This idea was conjectured independently by Grofman (1981). The distinction between the majority alternative and the alternative most likely to be best has also been discussed by Baker (1975) and Urken and Traflet (1984).

14. The following argument was suggested by Bernard Grofman.

15. This property of Condorcet's method (which is known in the literature as the "median procedure $^{\prime \prime}$ ) was noted by Jacquet-Lagrèze (1969). For other results on the median procedure see Michaud and Marcotorchino (1978), Barthelemy and Monjardet (1981), Michaud (1985), and Barthelemy and McMorris (1986).

16. For example, if there are an even number of voters and every pairwise vote is a tie, then anonymity and neutrality imply that all linear orderings of the alternatives are equally valid. Thus $F$ must sometimes take on multiple values.

17. We require furthermore that if another ranking is approved by only one of the two groups, then it is not tied with a ranking that is approved by both of them.

18. Let $F$ satisfy the five conditions of the theorem. When there are exactly two alternatives, it follows that $F$ is simple majority rule (Young 1974). Now consider the case of more than two alternatives. Let $R$ be the social order. Local stability implies that whenever two alternatives are ranked one immediately above another in $R$, then their ordering remains invariant when $F$ is restricted to those two alternatives alone. Since $F$ is simple majority rule on every two alternatives, it follows that in the social order $R$ every alternative must defeat (or tie) the alternative ranked immediately below it. Furthermore, if the two alternatives are tied, then they may be switched to obtain a social ordering tied with $R$. It can be shown by induction on the number of alternatives that this property, together with neutrality, unanimity, and reinforcement implies that $F$ is Condorcet's ranking rule (Young and Levenglick 1978).

\section{References}

Arrow, Kenneth J. 1963. Social Choice and Individual Values. $2 \mathrm{~d}$ ed. New York: John Wiley.

Baker, Keith M. 1975. Condorcet. Chicago: University of Chicago Press.

Barthelemy, J. P., and F. R. McMorris. 1986. "The Median Procedure for n-Trees." Journal of Classification 3:329-34.

Barthelemy, J. P., and Bernard Monjardet. 1981. "The Median Procedure in Cluster Analysis and 


\section{American Political Science Review Vol. 82}

Social Choice Theory." Mathematical Social Sciences 1:235-67.

Batchelder, William, and N. J. Bershad. 1979. "The Statistical Analysis of a Thurstonian Model for Rating Chess Players." Journal of Mathematical Psychology 19:39-60.

Black, Duncan. 1958. The Theory of Committees and Elections. Cambridge: Cambridge University Press.

Borda, Jean Charles de. 1784. "Mémoire sur les Elections au Scrutin." In Histoire de L'Academie Royale des Sciences.

Condorcet, Marquis de. 1785. Essai sur l'application de l'analyse à la probabilité des décisions rendues d̀ la probabilité des voix. Paris: De l'imprimerie royale.

Fishburn, Peter C. 1973. The Theory of Social Choice. Princeton: Princeton University Press.

Gelfand, Alan, and Herbert Solomon. 1973. "A Study of Poisson's Models for Jury Verdicts in Criminal and Civil Trials." Journal of the American Statistical Association 68:271-78.

Good, I. J. 1955. "On the Marking of Chess Players." The Mathematical Gazette 39:292-96.

Grofman, Bernard. 1981. "When Is the Condorcet Winner the Condorcet Winner?" University of California, Irvine. Typescript.

Grofman, Bernard, and Scott Feld. 1988. "Rousseau's General Will: A Condorcetian Perspective." American Political Science Review 82: 567-76.

Grofman, Bernard, and Guillermo Owen, eds. 1986. Information Pooling and Group Decision Making. Greenwich, CT: JAI.

Grofman, Bernard, Guillermo Owen, and Scott Feld. 1983. "Thirteen Theorems in Search of the Truth." Theory and Decision 15:261-78.

Henry, Charles, ed. 1883. Correspondance Inedité de Condorcet et de Turgot 1770-1779. Paris: Charavay frères.

Jacquet-Lagrèze, E. 1969. "L'Agrégation des opinions individuelles." In Informatiques et sciences humaines, vol. 4.

Jech, Thomas. 1983. "The Ranking of Incomplete Tournaments: A Mathematician's Guide to Popular Sports." American Mathematical Monthly 90:246-66.

Kemeny, John. 1959. "Mathematics without Num- bers." Daedalus 88:571-91.

Kemeny, John, and Lawrence Snell. 1960. Mathematical Models in the Social Sciences. Boston: Ginn.

Kramer, Gerald. 1977. "A Dynamical Model of Political Equilibrium." Journal of Economic Theory 16:310-34.

Mascart, Jean. 1919. La Vie et les travaux du Chevalier Jean Charles de Borda. Paris: Rey.

Michaud, P. 1985. “Hommage à Condorcet (version integrale pour le bicentenaire de l'essai de Condorcet)." Etude F-094. Centre scientifique-IBM France, Paris.

Michaud, P., and J. F. Marcotorchino. 1978. "Optimization in Ordinal Data Analysis." Etude F-001. Centre Scientifique-IBM France, Paris.

Nash, John. 1950. "The Bargaining Problem." Econometrica 18:155-62.

Nitzan, S., and J. Paroush. 1982. "Optimal Decision Rules in Uncertain Dichotomous Situations." International Economic Review 23:289-97.

Poisson, Siméon-Denis. 1837. Recherches sur la probabilité des jugements en matière criminale et en matière civile, précédées des règles générales $d u$ calcul des probabilités. Paris: Bachelier.

Rousseau, Jean-Jacques. 1962. The Social Contract. Harmondsworth, England: Penguin.

Shapley, Lloyd S., and Bernard Grofman. 1984. "Optimizing Group Judgmental Accuracy in the Presence of Interdependencies." Public Choice 43:329-43.

Todhunter, Isaac. 1949. A History of the Mathematical Theory of Probability. New York: Chelsea.

Urken, Arnold B., and S. Traflet. 1984. "Optimal Jury Design." Jurimetrics 24:218-35.

Young, H. Peyton. 1974. "An Axiomatization of Borda's Rule." Journal of Economic Theory 9: 43-52.

Young, H. Peyton. 1986. "Optimal Ranking and Choice from Pairwise Comparisons." In Information Pooling and Group Decision Making, ed. Bernard Grofman and Guillermo Owen. Greenwich, CT: JAI.

Young, H. Peyton, and Arthur Levenglick. 1978. "A Consistent Extension of Condorcet's Election Principle." SIAM Journal on Applied Mathematics 35:285-300.

H. P. Young is Professor of Public Policy and Applied Mathematics, School of Public Affairs, University of Maryland, College Park, MD 20742. 